5-1-1996

\title{
Structure of a Bovine Thrombin-Hirudin 51-65 Complex Determined by a Combination of Molecular Replacement and Graphics. Incorporation of Known Structural Information in Molecular Replacement
}

Jacqueline Vitali

Cleveland State University, j.vitali@csuohio.edu

Philip D. Martin

Wayne State University

Follow this and additional works at: https://engagedscholarship.csuohio.edu/sciphysics_facpub

"ichael G. Malkowski

wayne state Universysycs Commons

How does access to this work benefit you? Let us know!

Gisplysplepts Statement

CQ 1996 International Union of Crystallography

Paul H. Iohnson

SRI International

Original Citation

Vitali, J., Martin, P.D., Malkowski, M.G., Olsen, C.M., Johnson, P.H. and Edwards, B.F.P. (1996) Crystal

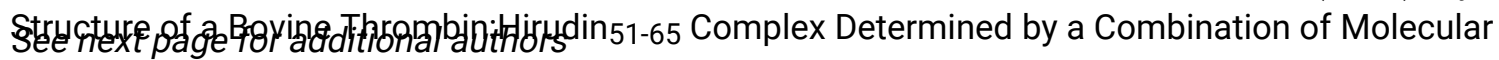
Replacement and Graphics. Incorporation of Known Structural Information in Molecular Replacement. Acta Crystallographica D52, 453-464.

Repository Citation

Vitali, Jacqueline; Martin, Philip D.; Malkowski, Michael G.; Olsen, Cris M.; Johnson, Paul H.; and Edwards, Brian F.P., "Structure of a Bovine Thrombin-Hirudin51-65 Complex Determined by a Combination of Molecular Replacement and Graphics. Incorporation of Known Structural Information in Molecular Replacement" (1996). Physics Faculty Publications. 181.

https://engagedscholarship.csuohio.edu/sciphysics_facpub/181

This Article is brought to you for free and open access by the Physics Department at EngagedScholarship@CSU. It has been accepted for inclusion in Physics Faculty Publications by an authorized administrator of EngagedScholarship@CSU. For more information, please contact library.es@csuohio.edu. 


\section{Authors}

Jacqueline Vitali, Philip D. Martin, Michael G. Malkowski, Cris M. Olsen, Paul H. Johnson, and Brian F.P. Edwards 


\title{
Structure of a Bovine Thrombin-Hirudin ${ }_{51-65}$ Complex Determined by a Combination of Molecular Replacement and Graphics. Incorporation of Known Structural Information in Molecular Replacement
}

\author{
Jacqueline Vitali,${ }^{a *}$ Philip D. Martin, ${ }^{a}$ Michael G. Malkowski,${ }^{a}$ Cris M. Olsen,${ }^{b}$ Paul. H. Johnson ${ }^{c} \dagger$ And \\ BRIAN F. P. EDWARDS ${ }^{a *}$
}

${ }^{a}$ The Department of Biochemistry, Wayne State University, Detroit, MI 48201, USA, and the ${ }^{b}$ Bio-Organic and ${ }^{c}$ Cell and Molecular Biology Laboratories, SRI International, Menlo Park, CA 94025, USA. E-mail: bedward@cms.cc.wayne.edu

(Received 31 March 1995; accepted 5 January 1996)

\begin{abstract}
Crystals of the bovine thrombin-hirudin ${ }_{51-65}$ complex have space group $P 6_{1} 22$ with cell constants $a=116.4$, and $c=200.6 \AA$ and two thrombin molecules in the asymmetric unit. Only one thrombin molecule could be located by generalized molecular replacement; the second was fit visually as a rigid body to an improved electron-density difference map. The structure was refined to $R=0.192$ with two $B$ values per residue (main chain and side chain) at $3.2 \AA$. The polar interactions of the peptides with the exosite of thrombin show differences consistent with the known flexibility in the interactions of the C-terminal peptide of hirudin with thrombin. The hirudin peptide in complex 2 has a higher temperature factor as compared with peptide 1 which may be correlated partly with a larger number of short-range electrostatic interactions between peptide 1 and thrombin and partly with the fact that thrombin 2 is $\varepsilon$-thrombin which is cleaved at Thr149A near the peptide binding site. Later, using this structure as a test case, it was shown that the position for the second thrombin could also be determined by a novel modification of the molecular-replacement method in which the contribution of the known molecule is subtracted from the structure factors. This approach is facile and applicable to any crystal containing two or more macromolecules in the asymmetric unit in which some but not all of the molecules can be determined by molecular replacement.
\end{abstract}

\section{Introduction}

$\alpha$-Thrombin (E.C. 3.4.21.4), a trypsin-like serine protease that is activated by factor Xa cleavage of prothrombin, exhibits both procoagulant and anticoagulant activities in the blood-clotting cascade (reviewed by Mann, 1987; Davie, Fujikawa \& Kisiel,

† Current address: Berlex Biosciences, 15049 San Pablo Avenue, Richmond, CA 94804, USA.
1991). Free $\alpha$-thrombin triggers platelet clotting, generates fibrin and active factor XIII to stabilize the clot and activates clotting factors V, VIII and XI, which in turn generate more $\alpha$-thrombin (Fenton, 1986; Davie et al., 1991, and references therein). In contrast, $\alpha$-thrombin bound to thrombomodulin decelerates clotting by preferentially activating protein $\mathrm{C}$, which then proteolytically inactivates factors $\mathrm{Va}$ and VIIIa in the clotting cascade leading to thrombin (Esmon, 1987; Dittman \& Majerus, 1990). Thrombin also triggers the inflammation response to vascular damage by activating neutrophils and monocytes (reviewed by Coughlin, 1994).

Bovine $\alpha$-thrombin has an $A$ chain of 49 residues linked by a single disulfide bond to a $B$ chain of 259 residues (Magnusson, Petersen, Sottrup-Jensen \& Claeys, 1975; MacGillivray \& Davie, 1984; Walz, Hewett-Emmett \& Guillin, 1986) which is glycosylated at Asn60G. Human $\alpha$-thrombin has 13 fewer residues in the $A$ chain because of an additional cleavage during activation. The $B$ chain has a classic, trypsin-like active site with a catalytic triad comprised of His $57, \ddagger$ Asp 102 and Ser195 and an acidic residue, Asp189, at the bottom of the specificity pocket. In contrast with trypsin, however, access to the active site for macromolecular substrates is severely restricted in thrombin due to insertions of additional residues on either side of the active-site groove (Bode et al., 1989). Furthermore, thrombin has a unique secondary substrate-binding site, called the anion-binding site or exosite, which interacts with residues that are, relatively speaking, exceptionally far 'downstream' from the cleavage site (Fenton, 1981). Overall, human and bovine $\alpha$-thrombin have very similar properties despite nine sequence differences in the $A$ chain, and 34 sequence differences in the

$\ddagger$ The residue numbers for thrombin in this paper are assigned by homology with chymotrypsin (Bode et al., 1989). A suffix denotes an insertion in the thrombin sequence relative to that of chymotrypsin. Sequence numbers for hirudin are identified by the suffix ' $h$ '. The prime symbol $\left({ }^{\prime}\right)$ denotes a residue number in a symmetry-related molecule. 
B chain (Butkowski, Elion, Downing \& Mann, 1977; Degan, MacGillivray \& Davie, 1983; Walz et al., 1986).

Hirudin, an extremely potent inhibitor of clotting that is secreted by leeches (Markwardt, 1970), binds to bovine thrombin with a dissociation constant of $3.0 \mathrm{pM}$ and to human thrombin with a dissociation constant of $0.3 \mathrm{pM}$ (Dodt, Konler \& Baici, 1988; Dodt, Kohler, Schmitz \& Wilhelm, 1990). It has 65 residues, including a sulfated tyrosine at position 63 . The solution structure of free hirudin determined by NMR methods (Clore, Sukumaran, Nilges, Zarbock \& Gronenborn, 1987; Folkers et al., 1989; Haruyama \& Wüthrich, 1989) has a compact amino-terminal domain of 49 residues followed by 16 residues that are disordered. Crystal structures of the hirudin complex with bovine thrombin (Vitali et al., 1992) and with human thrombin (Grütter et al., 1990; Rydel et al., 1990; Rydel, Tulinsky, Bode \& Huber, 1991) show the aminoterminal domain of hirudin sitting across the active site while the carboxyl-terminal domain stretches out to the exosite in an extended, but generally well defined conformation.

The hirudin carboxyl-terminal peptide either alone (Maraganore, Chao, Joseph, Jablonski \& Ramachandran, 1989; DiMaio et al., 1990) or linked to an activesite ligand (Maraganore, Bourdon, Jablonski, Ramachandran \& Fenton, 1990) has been investigated as a synthetic analog of hirudin. Alone, the peptide binds only to the exosite where it inhibits fibrinogen binding and thus clotting but does not inhibit hydrolysis of small synthetic substrates of thrombin at the active site ( $\mathrm{Di}$ Maio et al., 1990; Maraganore et al., 1989). Inhibitors containing an active-site ligand linked to a C-terminal fragment of hirudin block both the active site and the exosite (Bourdon, Jablonski, Chao \& Maraganore, 1991; Yue et al., 1992). Carboxyl-terminal fragments of hirudin are more effective inhibitors of human as compared to bovine $\alpha$-thrombin by an order of magnitude (Maraganore et al., 1989; Di Maio et al., 1990).

Several structures of complexes of human $\alpha$-thrombin with inhibitors containing a C-terminal hirudin peptide in the exosite have been determined (Banner \& Hadvary, 1991; Skrzypczak-Jankun et al., 1991; Qiu et al., 1992; Stubbs et al., 1992; Maryanoff et al., 1993; Priestle, Rahuel, Rink, Tones \& Grütter, 1993; Zdanov et al., 1993). The thrombin-hirudin ${ }_{51-65}$ structure reported here differs from previous studies by (1) incorporating bovine thrombin, which has a lower binding affinity for hirudin instead of human thrombin, (2) using a high ionic strength precipitant instead polyethylene glycol, and (3) having the hirudin peptide complexed with both $\alpha$-thrombin and $\varepsilon$ thrombin in the asymmetric unit. Furthermore, this crystal form, in space group $P 6_{1} 22$ which is a new space group for thrombin structures, was a challenging crystallographic problem in which one of the thrombins in the asymmetric unit could be located by molecular replacement but not the other. While this problem was solved using graphics, a facile hardy method that uses modified structure factors in the molecular-replacement calculations was also developed to solve the "case of the missing molecule' which both we and others have encountered multiple times (Zhang \& Matthews, 1994). The method is general and applicable to any crystal containing two or more macromolecules in the asymmetric unit in which some but not all of the molecules can be determined by molecular replacement.

\section{Materials and methods}

\subsection{Preparation of bovine thrombin and hirudin 51-65}

Thrombin was prepared as previously described (Martin, Kumar, Tsernoglou \& Edwards, 1983) and had approximately 2000 NIH clotting units per mg of protein. It was stored at $203 \mathrm{~K}$ in ammonium phosphate pH 6.5 before being set up for crystallization. Hirudin 51-65 was synthesized by solid-phase techniques on a Boc-Gln-O-resin; following synthesis, removal of the protecting groups and cleavage of the peptide from the resin, the peptide extracts were lyophilized and purified by preparative reverse-phase high-pressure liquid chromatography (Johnson et al., 1991).

\subsection{Crystallization}

Bovine $\alpha$-thrombin was brought to $295 \mathrm{~K}$ concentrated to $23.5 \mathrm{mg} \mathrm{m}^{-1}$ by centrifugation using Centriprep 30 (Amicon) at $3600 \mathrm{rev} \mathrm{min}^{-1}$ and exchanged into $0.25 \mathrm{M}$ ammonium phosphate $\mathrm{pH} 8.0$ using a Sephadex G-25 column. The complex with the inhibitor was prepared using a molar ratio of $1: 3$. The crystals were grown by the hanging-drop method at $295 \mathrm{~K}$ from reservoirs containing $39 \%$ saturated ammonium sulfate, $0.25 \mathrm{M}$ ammonium phosphate $\mathrm{pH} 8.0$ and $1 \%$ PEG 4000 . The drops initially contained $5 \mu \mathrm{l}$ reservoir solution and $5 \mu \mathrm{l}$ complex solution. The crystals are hexagonal, cell constants $a=116.4, c=200.6 \AA$, space group $P 6_{1} 22$ with two complexes per asymmetric unit.

\subsection{Data collection}

Intensity data were measured at $295 \mathrm{~K}$ on the largest crystal (approximate dimensions $0.40 \times 0.20$ $\times 0.20 \mathrm{~mm}$ ) using a Siemens area detector with a three-circle goniostat, a Rigaku RU200H rotatinganode X-ray source and Supper graphite monochromator. The $\mathrm{Cu} K \alpha$ radiation was generated at $40 \mathrm{kV}$ and $70 \mathrm{~mA}$. The crystal-to-detector distance was $25 \mathrm{~cm}$ and the swing angle $2 \theta$ was $16^{\circ}$. One 'sweep' only was made covering $110^{\circ}$ in $\omega$ with 440 frames of data. Each frame was measured for $10 \mathrm{~min}$ to ensure that it contained at least 1.5 million total counts. A total of 31897 
measurements with $I>0$ were merged, scaled and averaged with the XENGEN package (Howard et al., $1987)$ to give 11300 unique reflections $(80 \%$ complete to $3.2 \AA$ ). The overall, unweighted, absolute $R_{\text {sym }}$ was 0.126 . The completeness and average $I / \sigma$ of the data for shells of increasing resolution were $89 \%$ and 14.3 for $\infty-5.8 \AA$. $92 \%$ and 8.2 for $5.8-4.6 \AA ; 90 \%$ and 6.3 for $4.6-4.0 \AA$; $86 \%$ and 4.1 for $4.0-3.7 \AA ; 82 \%$ and 2.8 for $3.7-3.4 \AA$; and $41 \%$ and 2.0 for $3.4-3.2 \AA$.

\subsection{Structure solution}

The orientation and position of one of the two thrombins (thrombin 1) was determined by the generalized molecular-replacement method using X-PLOR (Brünger, 1988, 1990). The probe used in these calculations was bovine $\alpha$-thrombin in the complex with hirudin (Vitali et al., 1992). Residues 1H-1C and residue 15 of the $A$ chain, the $\gamma$-autolysis loop residues 147-149D and residues 245-247 of the $B$ chain were not part of the model. These segments are flexible and are likely to differ between the present structure and bovine thrombin-hirudin. The real-space Patterson search was calculated using data in the resolution range 15.0-4.0 $\AA$ and a shell of integration $5.0-24.0 \AA$. The selected peaks of the rotation function were subsequently Patterson correlation (PC) refined in 15 steps using data $15.0-4.0 \AA$. The data used in the translation searches were in the resolution range 7.0-4.0 $\AA$, and the grid interval was $1.28 \AA$ along $a$ and $b$, and $1.20 \AA$ along $c$. All molecular-replacement calculations used data with $|F|>4 \sigma$.

All efforts to locate thrombin molecule 2 by the generalized molecular-replacement method proved unsuccessful. However, the $\mathrm{C}_{\alpha}$ backbone of thrombin was fitted as a rigid body by visual inspection of an electron-density difference map computed with all available data in the resolution range $20.0-3.2 \AA$, and with phases (calculated from molecule 1) improved by solvent flattening, histogram matching and Sayre's equation using SQUASH (Zhang, 1993). This solution was confirmed by refinement.

\subsection{Refinement}

The parameters of Engh \& Huber (1991) and the standard input files of $X-P L O R$ for rigid-body, positional, simulated-annealing, and $B$-factor refinement were used. $C_{\alpha}$ atoms related by non-crystallographic symmetry were weakly restrained in their average positions throughout the refinement using an effective energy constant of $100 \mathrm{kcal} \mathrm{mol}^{-1} \AA^{-2}$. In order to ensure good geometry, at most stages of the refinement, weights equal to half the weight from the checkstage were used. At the early stages of the analysis, the program GPRLSA (Furey, Wang \& Sax, 1982) was used for the refinement of the positional and thermal parameters. At all stages, the data used in the refinement were in the resolution range 7.0-3.2 with $|F|>2 \sigma$. All graphics rebuilding of the model was carried out with the program TOM/FRODO (Cambillau \& Horjales, 1987; Jones, 1985).

\subsection{Incorporation of known structural information in molecular replacement}

Four electron-density difference maps were calculated with phases from thrombin molecule 1 as follows: (I) an unmodified $\left|F_{o}\right|-\left|F_{c}\right|$ electron-density map, (II) an $\left|F_{o}\right|-\left|F_{c}\right|$ electron-density map with figures of merit and phases improved by solvent flattening, histogram matching and Sayre's equation using SQUASH, (III) a $2\left|F_{o}\right|-\left|F_{c}\right|$ electron-density map and (IV) a $2\left|F_{o}\right|-\left|F_{c}\right|$ electron-density map with figures of merit and phases improved by solvent flattening, histogram matching, and Sayre's equation using SQUASH. The electron density within $2.0 \AA$ of the atomic positions of thrombin 1 was then set to zero in maps (III) and (IV) (RHOMOD) program; P. Martin, unpublished work). These four 'thombin-1 subtracted' maps were inverted with programs from W. Furey Jr (University of Pittsburgh) to generate structure factors which corresponded to the uninterpreted regions of the unit cell. The modified structure factors $(15.0-4.0 \AA)$ were used for rotation function, $\mathrm{PC}$ and translation-function calculations in $X-P L O R$. The rotation function was calculated with a 5.0-24.0 A shell of integration and PC refinement was carried out in 15 steps.

\subsection{Structure analysis}

Hydrogen bonds were calculated in QUANTA (Biosym/MSI, San Diego, CA, USA) using as criteria a distance of $3.5 \AA$ or less between the donor and acceptor atoms and a value of $90^{\circ}$ or more for the angles centered at these atoms. A maximum distance of $4.0 \AA$ was used for hydrophobic contacts and salt links. Coordinate sets 1HRT for bovine thrombin-hirudin (Vitali et al., 1992) and 1HAH for human thrombinhirugen* (Skrzypczak-Jankun et al., 1991; Vijayalakshmi, Padmanabhan, Mann \& Tulinsky, 1994) from the Protein Data Bank (Abola, Bernstein, Bryant, Koetzle \& Wang, 1987) were used for comparative analysis. All overlaps were calculated with the program ALIGN (Satow, Cohen, Padlan \& Davies, 1986).

\section{Results and discussion}

\subsection{Structure solution}

The orientation of thrombin 1 was weakly indicated by the rotation function. It corresponded to the top selected peak, at $\left(\theta_{1}, \theta_{2}, \theta_{3}\right)=\left(265.0,65.0,47.5^{\circ}\right)$ but was only 1.05 times greater than the highest noise peak.

* Hirugen is hirudin residues $53 \mathrm{~h}-65 \mathrm{~h}$ sulfated at Tyr63h. 
After PC refinement, the signal-to-noise ratio of this peak substantially improved and its correlation coefficient of 0.034 was 1.48 times greater than the highest PC value corresponding to an error peak. The top peak in the translation search for space group $P 6_{1} 22$, which had a correlation coefficient of 0.306 , corresponded to the position of thrombin 1 in the unit cell at $(0.400$, $0.200,0.452$ ), and was 1.62 times greater than the next highest independent peak. The determination of the space group was unambiguous. The top peaks in the translation searches for space groups $P 6_{5} 22, P 6_{2} 22$, $P 6_{4} 22, P 6_{3} 22$ and $P 622$, which had correlation cocfficients of $0.138,0.116,0.143,0.122$ and 0.116 , respectively, were much smaller. Refinement of the rigid-body parameters of thrombin 1 with $X-P L O R$ followed by refinement of the positional parameters with GPRLSA gave a crystallographic $R$ factor of 0.356 , indicating that the molecular-replacement solution for thrombin 1 was correct.

Residues 154-163, 129C-139, and 196-206, corresponding to part of one of the two $\beta$-barrels of thrombin 2 , the connecting segments between $\beta$-strands 164-179 and $97-100$, and residues 231-239 containing part of a helix of thrombin were recognized in the SQUASH electron-density difference map and were used as a guide in fitting the $\mathrm{C}_{\alpha}$ backbone of thrombin as a rigid body to the density of this map (Fig. 1). Refinement of the rigid-body parameters of both thrombins with $X-P L O R$, followed by refinement of their positional and thermal parameters with GPRLSA reduced the $R$ factor to 0.211 , indicating that the graphics solution for thrombin 2 was correct.

\subsection{Refinement}

Electron density for residues $56 \mathrm{~h}-60 \mathrm{~h}$ of peptide 1 bound to thrombin 1 and for residues $56 \mathrm{~h}$ and $57 \mathrm{~h}$ of peptide 2 bound to thrombin 2 at positions corresponding to those in the bovine and human thrombin-hirudin complexes was apparent in the $2\left|F_{o}\right|-\left|F_{c}\right|$ electron-density map phased with the two thrombin molecules. The remaining residues of both peptides had fragmented electron density. Because the density for the carboxyl-terminal turn was also weak in our bovine thrombin-hirudin complex, the human thrombin-hirudin complex was superimposed on both thrombin molecules and residues $55 \mathrm{~h}-65 \mathrm{~h}$ from human thrombin-hirudin were included in our model for both complexes. After refinement of the rigid-body parameters of both peptides, seven cycles of positional and $B$-factor refinement, simulated annealing and five further cycles of positional and $B$-factor refinement followed, all alternating with manual rebuilding of the model. The final $R$ value

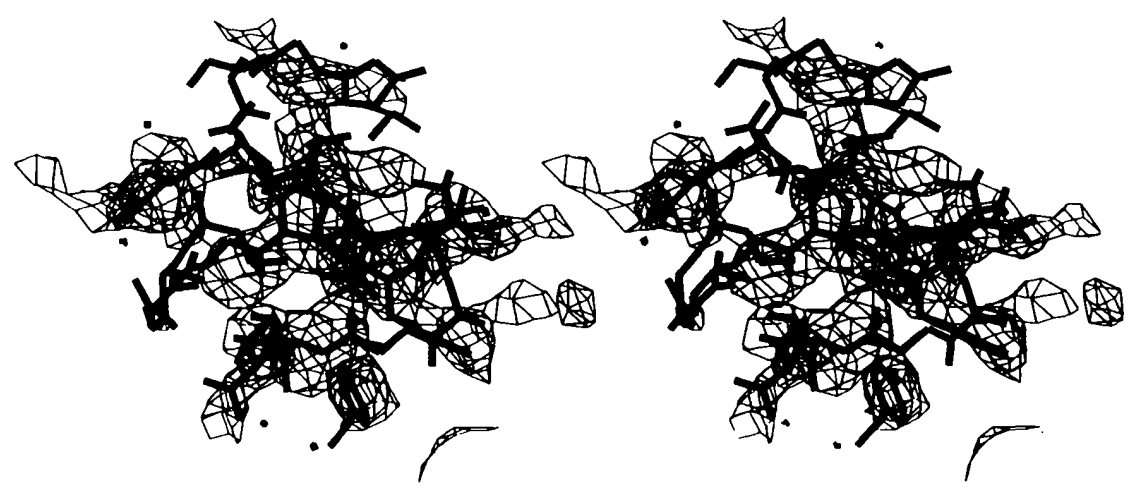

(a)

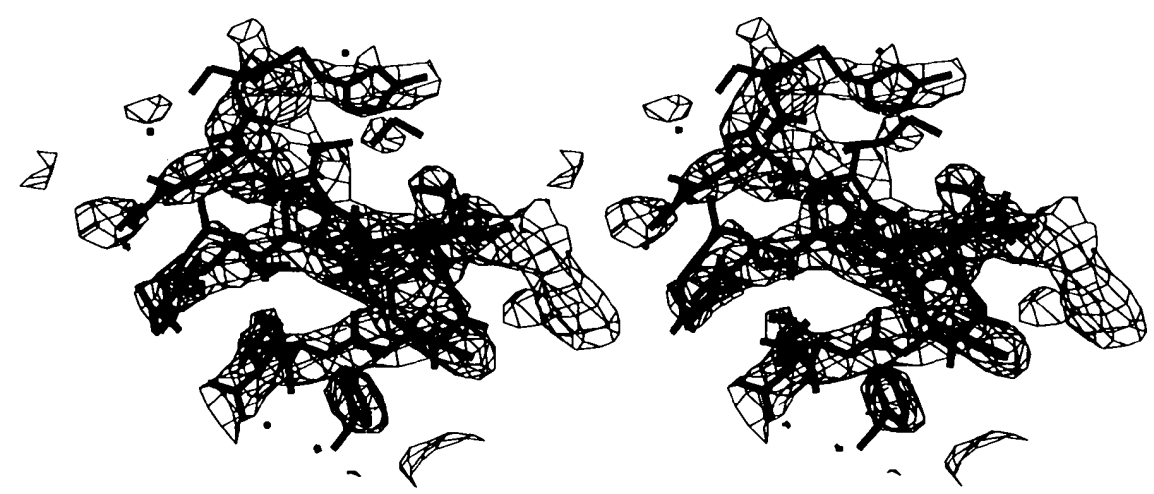

(b)
Fig. 1. Stereoviews of the electron density around Pro198. (a) The SQUASH electron-density difference map from which thrombin 2 was determined contoured at 1.3o. (b) The same view in the final $2\left|F_{o}\right|-\left|F_{c}\right|$ electron-density map contoured at $1.7 \sigma$. 
of the structure is 0.192 with an r.m.s. deviation of $0.009 \AA$ for bond lengths and $1.88^{\circ}$ for bond angles. Because of the moderate resolution of this analysis, two 'group' $B$ values were used for each residue in the final model, one for the main-chain atoms and one for the side-chain atoms. The variation of the $R$ value as a function of resolution is given in Table 1. The mean error in the coordinates estimated from SIGMAA is $0.5 \AA$ (Read, 1986). When analyzed by PROCHECK (Laskowski, MacArthur \& Thornton, 1994), $96.9 \%$ of the residues fell within the allowed regions of a Ramachandran plot, $2.1 \%$ were in the
Table 1. Variation of the $R$ value as a function of resolution

$\begin{array}{cccc}D_{\min }(\dot{\mathrm{A}})^{*} & \text { No. of reflections } & R_{\text {shell }} & R_{\text {sphere }} \\ 5.40 & 1264 & 0.223 & 0.223 \\ 4.67 & 1360 & 0.173 & 0.195 \\ 4.22 & 1292 & 0.167 & 0.185 \\ 3.91 & 1192 & 0.174 & 0.182 \\ 3.67 & 1096 & 0.178 & 0.182 \\ 3.49 & 1103 & 0.216 & 0.186 \\ 3.33 & 937 & 0.221 & 0.189 \\ 3.20 & 415 & 0.253 & 0.192\end{array}$

* Reflections beyond $7.0 \dot{\mathrm{A}}$ were not included.

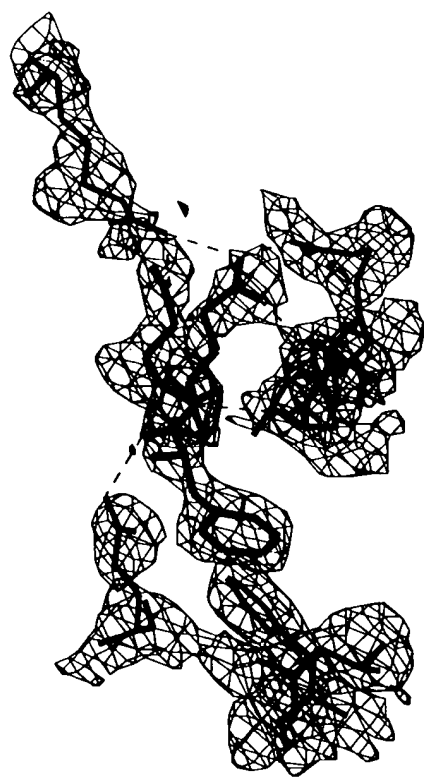

(a)

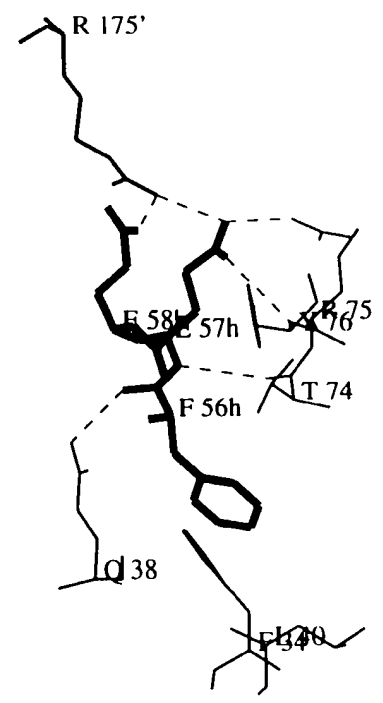

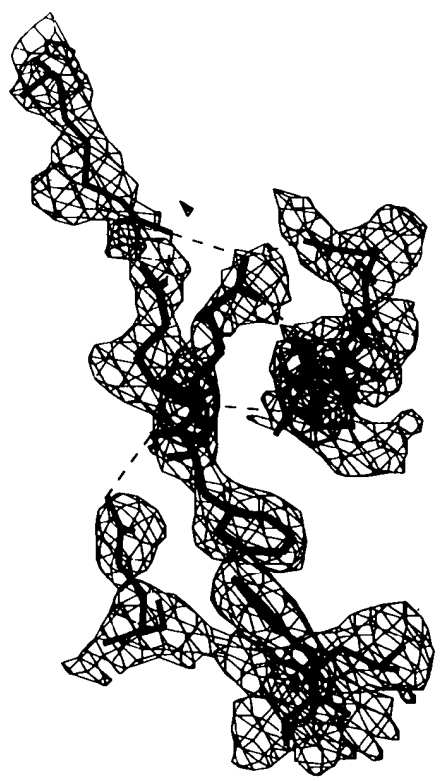

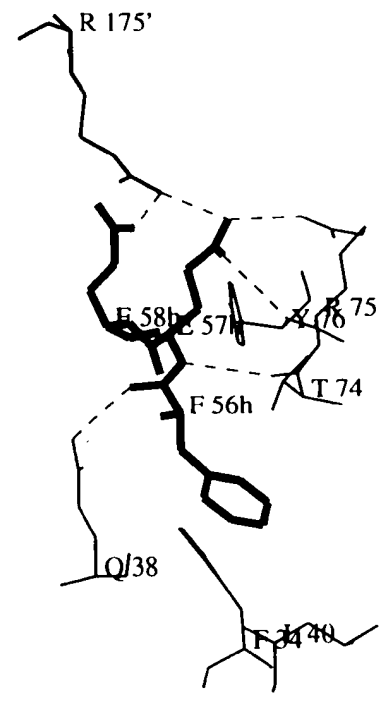

(b)

Fig. 2. Stereoviews of the interactions of residues Phe56hGlu58h of peptide 1 with thrombin. (a) The final $2\left|F_{o}\right|-\left|F_{c}\right|$ electron-density within $2.0 \dot{\mathrm{A}}$ of peptide 1 contoured at $1.0 \sigma$. Thick lines are used for bonds in peptide 1; medium weight lines for bonds in thrombin. (b) The skeletal representation of the interactions. Thick lines are used for bonds in peptide 1; thin lines for bonds in thrombin. 
generously allowed regions and $1 \%$ were in disallowed areas. Other measures of the stereochemistry, including the standard deviations for the $\omega$ angles $\left(1.5^{\circ}\right), \zeta$ angles $\left(1.9^{\circ}\right)$, and pooled $\chi_{1}$ angles $\left(19.5^{\circ}\right)$ are better than the average values for other structures at a similar resolution. Peptide 2 has weaker electron density and higher temperature factors (average $B=74 \AA^{2}$ ) as compared to peptide 1 (average $B=56 \AA^{2}$ ), even though thrombin molecules 1 and 2 have similar average temperature factors of 20 and $25 \AA^{2}$. The fit of the refined model to the electron density is shown in Figs. 1, 2(a) and $3(a)$.

\subsection{The failure of the generalized molecular-replace- ment method to determine thrombin 2}

The correct orientation of thrombin 2 has Euler angles $110.4,79.7$, and $7.5^{\circ}$ in the asymmetric unit of the rotation function. The 57 th selected peak of the

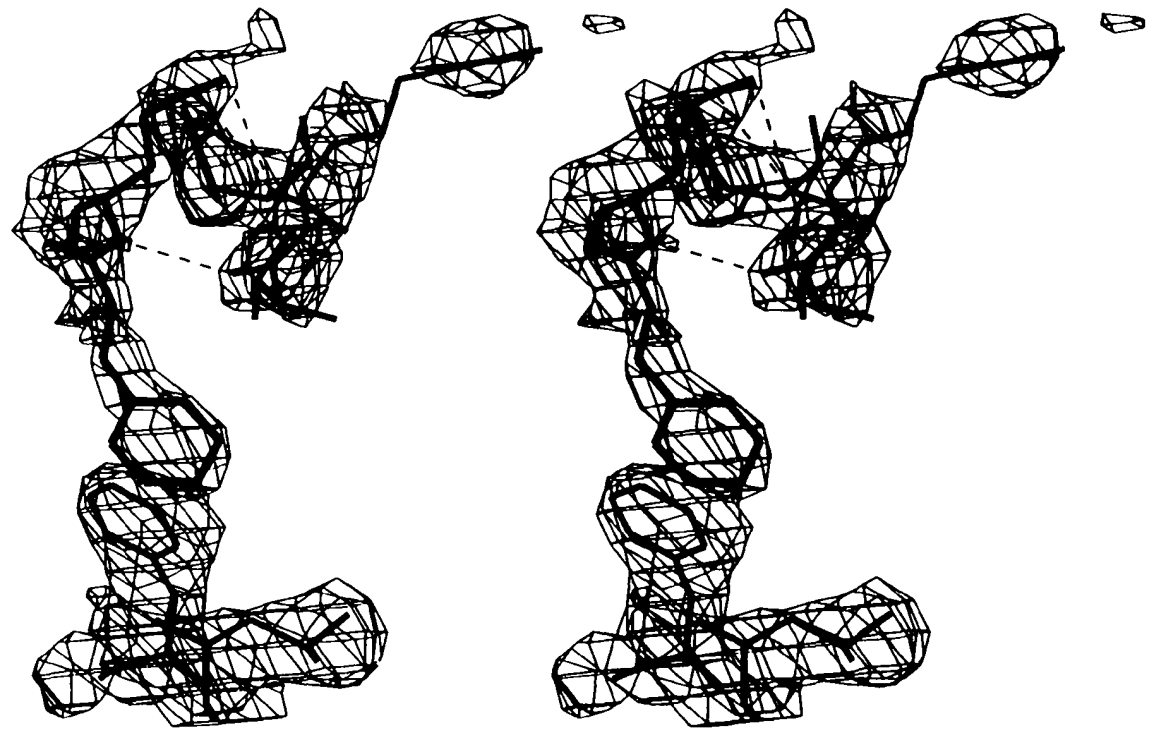

(a)

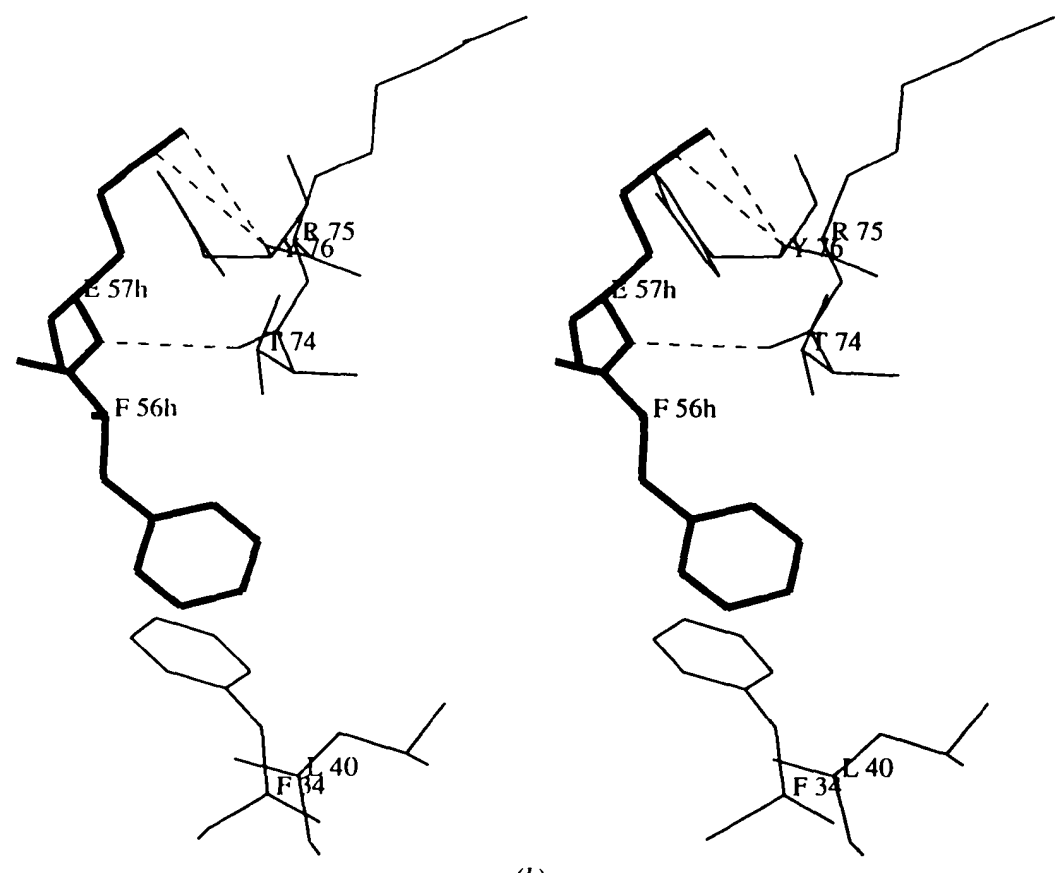

(b)
Fig. 3. Stereoviews of the interactions of residues Phe56h-Glu57h of peptide 2 with thrombin. (a) The refined $2\left|F_{o}\right|-\left|F_{c}\right|$ electron density within $2.0 \dot{A}$ of peptide 2 contoured at $0.9 \sigma$. Thick lines are used for bonds in peptide 2; medium-weight lines for bonds in thrombin. (b) The skeletal representation of the interactions. Thick lines are used for bonds in peptide 1; thin lines for bonds in thrombin. 
Table 2. Comparison of the effect of the modifications of the structure factors on determining thrombin 2 by molecular replacement

The calculations reported were carried out using $15.0-4.0 \dot{A}$ data, unless stated otherwise, and a 5.0-24.0 $\dot{A}$ shell of integration for the rotation function. The signal to noise $(S / N)$ is calculated as the peakheight ratio of the correct peak to the highest error peak. The deviation of an orientation from the correct orientation of thrombin 2 is measured by the overall rotation $\delta(X-P L O R)$ that relates the two orientations. The Eulerian angles $\theta_{1}, \theta_{2}, \theta_{3}$ are according to the convention of Rossmann \& Blow (1962), and $X, Y, Z$ are fractional coordinates. All angles are in degrees.

(a) Rotation search

$\begin{array}{lcccccc}\text { Modification } & \theta_{1} & \theta_{2} & \theta_{3} & \delta & \text { Rank } & S / N \\ \text { None } & 112.5 & 77.5 & 0.0 & 7.7 & 57 & 0.94 \\ \text { (I) } & 110.0 & 82.5 & 5.0 & 3.8 & 26 & 0.89 \\ \text { (II) } & 110.0 & 85.0 & 7.5 & 5.3 & 18 & 0.91 \\ \text { (III) } & 112.5 & 77.5 & 5.0 & 3.7 & 3 & 0.98 \\ \text { (IV) } & 112.5 & 77.5 & 5.0 & 3.7 & 1 & 1.03\end{array}$

(b) Patterson correlation refinement

\begin{tabular}{lcrrrrrc} 
Modification & $\theta_{1}$ & $\theta_{2}$ & \multicolumn{1}{c}{$\theta_{3}$} & $\delta$ & Rank & $S / N$ & $\begin{array}{c}\text { Correlation } \\
\text { coefficient }\end{array}$ \\
None & 114.1 & 73.2 & -1.6 & 11.1 & 17 & 0.61 & 0.014 \\
(I) & 110.7 & 78.9 & 6.6 & 1.2 & 6 & 0.60 & 0.014 \\
(II) & 110.7 & 77.7 & 6.4 & 2.3 & 3 & 0.98 & 0.017 \\
(III) & 110.6 & 78.4 & 6.4 & 1.6 & 1 & 1.03 & 0.020 \\
(IV) & 110.9 & 78.0 & 6.6 & 1.9 & 1 & 1.14 & 0.022
\end{tabular}

(c) Translation search*

$\begin{array}{lcccccc}\text { Modification } & X & Y & Z & \text { Rank } & S / N & \begin{array}{c}\text { Correlation } \\ \text { coefficient }\end{array} \\ \text { None } & \text { Failed } & & & & & \\ \text { (I) } & 0.122 & 0.456 & 0.428 & 1 & 1.41 & 0.208 \\ \text { (II) } & 0.122 & 0.444 & 0.416 & 1 & 1.29 & 0.189 \\ \text { (III) } & 0.122 & 0.456 & 0.428 & 1 & 1.48 & 0.270 \\ \text { (IV) } & 0.122 & 0.456 & 0.428 & 1 & 1.45 & 0.240\end{array}$

* The translation search was carried out using 7.0-4.0 $\dot{\mathrm{A}}$ data

rotation function had Euler angles of $112.5,77.5$ and $0.0^{\circ}$ that were close to the correct orientation. Its ratio to the rotation-function value of the top noise peak was 0.94. After 15 steps of PC refinement, this orientation had Euler angles of $114.1,73.2,-1.6^{\circ}$ and a correlation coefficient of 0.014 , which placed it 17 th in the list of the PC-refined selected peaks. Its ratio to the top error peak was only 0.61 . The low signal-tonoise was the reason that this peak was overlooked during structure determination. Moreover, a threedimensional translation search carried out for the purpose of this analysis with this $\mathrm{PC}$ refined orientation using 7.0-4.0 $\mathrm{A}$ data was not successful. Not one of the 100 top peaks of the translation function corresponded to the correct position. Translation searches are very sensitive to errors in the orientation and the PC refinement moved the molecule further away from the correct orientation. The PC-refined angles, (114.1, $\left.73.2,-1.6^{\circ}\right)$, differed by an overall rotation $(\delta)$ of $11.1^{\circ}$ from the correct orientation. The corresponding figure for the rotation-function angles was 7.7 . When the calculations were repeated with $8.0-5.0 \AA$ data and a 5.0-24.0 $\AA$ shell of integration for the rotation function, the closest orientation to the correct solution ranked 29th in the rotation function and at the top but equal to the highest noise peak after PC refinement. It did not give the correct position in the top 100 peaks of the translation function.

Even when starting with the accurately oriented search model, PC refinement moves thrombin 2 away from the correct orientation. After ten steps of PC refinement, the refined angles deviate from the correct orientation by rotations of $4.6^{\circ}$ for $15.0-4.0 \AA$ data and 6.5 for $8.0-5.0 \AA$ data. The sensitivity of the translation function to the accuracy of the orientation is illustrated by carrying out translation searches of the model at the correct orientation without and with PC refinement. Without $\mathrm{PC}$ refinement, these searches gave high values of the correlation coefficient of 0.239 and 0.253 for $15.0-4.0$ and $8.0-5.0 \AA$ data, respectively, at the correct position. The corresponding ratios to the top noise peaks were 1.45 and 1.18. After ten steps of PC refinement, the correlation coefficient dropped to 0.170 and the ratio to the top error peak was reduced to 1.20 for 15.0-4.0 $\AA$ data. For 8.0-5.0 $\AA$ data, not one of the top 50 peaks of the translation function corresponded to the correct position.

\subsection{Incorporation of known structural information in molecular replacement}

Subtracting the contribution of thrombin 1 from the structure factors as described in Methods substantially improves the PC refinement and the signal-to-noise ratios in the molecular-replacement calculations (Table 2 ). For the rotation function, the peak corresponding to thrombin 2 ranked significantly higher in the list of the selected peaks for all modifications as compared to the unmodified structure factors in the order (IV) $>$ (III) $>$ (II) $>$ (I). The signal-to-noise ratio improved only with modifications (III) and (IV) and only in the latter was the correct peak the highest in the list.

The correct orientation for thrombin 2 was the top peak in the PC refinement for modifications (III) and (IV) with (IV) having the highest signal-to-noise ratio. For modification (II), the peak corresponding to thrombin 2 was one of the three top peaks in the list, all with equal correlation coefficient. For modification (I), the signal-to-noise ratio did not improve as compared to the unmodified structure factors but the improvement in position from 17th to 6th meant there were fewer error peaks to be examined. It is especially cogent that PC refinement with all four modifications brought thrombin 2 very close to the correct orientation, differing only by 1.2 to $2.3^{\circ}$ ( $\delta$ in Table 2 ) because the 
improvement allowed the translation-function calculations to succeed.

Because the orientations were so accurate after PC refinement, the translation searches, which failed abysmally for the unmodified structure factors, succeeded for all modifications. The correct placement corresponded to the top peak in all searches with a high signal-to-noise ratio ranging from 1.29 to 1.47 . There appears to be no correlation between the signal-to-noise ratio and the specific modification. In summary, all four modifications of the structure factors substantially improve the results of the molecular-replacement calculations with modification (IV) $\simeq$ (III) $>$ (II) $>$ (I) . The more the structure factors depend on the unknown molecule, the more effective the modification.

Zhang \& Matthews (1994) recently incorporated known structural information in the rotation and translation functions using 'addition' and 'subtraction' strategies. With 'addition' strategy the structure factors of the known part of the structure are added to those of the search model. This procedure is most effective in the context of the translation function because it brings the structure factors calculated from the search model closer to those observed. With the 'subtraction' strategy, the Patterson function of the known component is subtracted from the 'observed' Patterson. This approach is found to be most effective in the context of the rotation function because it eliminates peaks that are irrelevant to the desired solution. Our approach to the problem, which was developed independently of theirs, is conceptually similar to their 'subtraction' strategy but computationally distinct, involving modifications of the structure factors external to the molecular-replacement programs as compared to modifying the rotation and translation functions, and can be used in conjunction with any of the available molecular-replacement packages without modifications to the programs. The results are similar to those obtained with the modified rotation function incorporating subtraction strategy, namely, a marked improvement in the rotational error as well as in the significance of the solution.

\subsection{Structure of the complexes}

The final model consists of residues $1 \mathrm{H}$ to 247 of both thrombin molecules with two sequential $\mathrm{N}$-acetylglucosamines linked at Asn60G of thrombin 1, one $\mathrm{N}$-acetylglucosamine linked at Asn60G of thrombin 2 and the two peptides. Except for a few residues, the two thrombin molecules are well defined in the electrondensity maps for residues $1 \mathrm{C}-14 \mathrm{~K}$ of the $A$ chain and residues 16-243 of the $B$ chain. Crystals of bovine thrombin commonly incorporate both $\alpha$-thrombin and a proteolytic derivative, $\varepsilon$-thrombin, which is cleaved in the $\gamma$-autolysis loop (residues 145-150) at Thr149A (Martin et al., 1992). This loop contains the eponymous human $\gamma$-thrombin cleavage site at Lys149E that is a glutamate residue in bovine thrombin and thus not cleaved. In complex 1 , the electron density for this mobile loop is present at $0.9 \sigma$ except for the amide $\mathrm{N}$ atom of Val149C in the main chain and some atoms in the side chains of Trp148, Thr149A and Val149C. In complex 2 , the main-chain density for residues Glu 146Thr149A is continuous, as is the density for Ser149B through Glu149E, but the $\mathrm{C}_{\alpha}$ atoms of Thr149A and Ser149B are $21 \AA$ apart. These observations imply that thrombin 1 is primarily $\alpha$-thrombin and thrombin 2 is primarily $\varepsilon$-thrombin.

The first 11 residues of peptide 1 bound to $\alpha$-thrombin, namely His $51 \mathrm{~h}-\mathrm{Glu} 61 \mathrm{~h}$, have reasonable electron density although His51h and Gly54h have some atoms out of density. The final four carboxyl-terminal residues are less certain, with Tyr63h being the only residue with reasonable density for all main-chain and side-chain atoms. Peptide 2 is bound nuch less securely to $\varepsilon$-thrombin than is peptide 1 to $\alpha$-thrombin. Five of the first seven residues in peptide 2, namely His $51 \mathrm{~h}$, Asn52h, and Asp55h-Glu57h have reasonable density but the remaining eight carboxyl-terminal residues, Glu58h-Gln65h, are effectively disordered with continuous density only for Glu62h, which has crystal packing contacts, and Tyr63h.

The two complexes, which were restrained to be similar during refinement, have an r.m.s. difference of $0.18 \AA$ between corresponding $C_{\alpha}$ atoms. They are related by an overall rotation of $172.8^{\circ}$ (Fig. 4). Compared to the bovine $\alpha$-thrombin-hirudin complex, the r.m.s. deviations in $\mathrm{C}_{\alpha}$ positions are $0.56 \AA$ for complex 1 and $0.59 \AA$ from complex 2 . Compared to the human $\alpha$-thrombin-hirugen structure, the corresponding r.m.s. deviations are 0.46 and $0.48 \AA$. Because residues Pro186-Arg187 of thrombin 1 and residues Lys185-Arg187 of thrombin 2 are involved in intermolecular interactions, Gly186A-Gly186C in complex 1 and Gly186C in complex 2 differ from bovine thrombin-hirudin by 1.1-1.9 $\dot{\mathrm{A}}$. Also, in both complexes, Gly $186 \mathrm{C}$ shows the largest deviations from the human thrombin-hirugen structure $(2.0 \AA)$.

Both peptides bind to their corresponding thrombins in the fibrinogen exosite, between two surface loops formed by residues Lys70-Phe80 on one side and Phe34-Leu41 on the other, as the corresponding segments do in the hirudin-thrombin complexes (Rydel et al., 1990, 1991; Grütter et al., 1990; Vitali et al., 1992) and in other thrombin-inhibitor complexes containing hirudin C-terminal peptide fragments (Skrzypczak-Jankun et al., 1991; Qiu et al., 1992; Maryanoff et al., 1993; Wu et al., 1993; Zdanov et al., 1993; Priestle et al., 1993; Stubbs et al., 1992; Banner \& Hadvary, 1991). The total solvent-accessible surface area buried in the interaction between residues $51 \mathrm{~h}-65 \mathrm{~h}$ of the peptide and thrombin is $1667 \AA^{2}$ in complex 1 and $1689 \AA^{2}$ in 
complex 2, which is slightly less than one half of the surface area buried in the interaction between intact hirudin and bovine thrombin (Vitali et al., 1992). Because of the reduced interaction surface, the $K_{i}$ for carboxyl-terminal peptides of hirudin is in the micromolar range (Maraganore et al., 1990) as opposed to the picomolar range for hirudin.

At physiological ionic strengths, the match of the negatively charged carboxyl terminus of hirudin to the positively charged exosite of thrombin drives the formation of this initial complex between the two regions (reviewed by Stone, Betz, Parry, Jackman \& Hofsteenge, 1993). In the structures determined here, complex 1 (Fig. 2b) is stabilized by three hydrogen bonds and four salt bridges, of which three involve a symmetry-related thrombin molecule, while complex 2 (Fig. $3 b$ ) is stabilized by just three hydrogen bonds (Table 3).

The electrostatic interactions of the two peptides with thrombin have some similarities but also differ from each other, from bovine thrombin-hirudin (Vitali et al., 1992) and from human thrombin-hirugen (SkrzypczakJankun et al., 1991). Both complexes have the hydrogen bond between the amide of Glu57h and the carbonyl $\mathrm{O}$ atom of Thr 74 that is conserved among all the hirudinhirugen complexes (Figs. $2 b$ and $3 b$ ). The two complexes also have a hydrogen bond between the side-chain OE1 of Glu57h and the amide of Tyr76 that is more weakly conserved. It is present in bovine thrombin-hirudin as a 'long' hydrogen bond but absent in human thrombin-hirugen.

The side chain of Glu57h also exemplifies the differences among the structures. In complex 1 , the OE2 atom forms salt bridges with Arg75 and Arg175' in a symmetry-related complex; in complex 2 it forms a hydrogen bond to the amide of Tyr76; and in human thrombin-hirugen it makes a salt link with $\operatorname{Arg} 75^{\prime}$ in a symmetry-related complex. In bovine thrombin-hirudin Glu57h OE2 has no partner but instead the OE1 atom makes a salt bridge with Arg75 as well as the conserved hydrogen bond mentioned above with Tyr76. Another striking example involves the side chain of Gln38 which forms a hydrogen bond to the carbonyl $\mathrm{O}$ atom of Phe56h in complex 1 but has no interactions in complex 2. It is hydrogen bonded to the amide of Phe $56 \mathrm{~h}$ in bovine thrombin-hirudin and to the carbonyl $\mathrm{O}$ atom of Phe57h in human thrombin-hirugen. These and other observed differences among the various hirudin and hirudin peptide complexes are consistent with the conclusion that there is some flexibility in the binding of the C-terminal peptide of hirudin to thrombin (Vitali et al., 1992) and with the idea that the function of the charged residues is to facilitate long-range alignment of the molecules and not for specific short-range interactions (Karshikov, Bode, Tulinsky \& Stone, 1992).

Mutagenesis has identified Glu57h in hirudin as the most important charged residue for binding thrombin, although Asp55h, Glu58h, Glu61h and Glu62h all
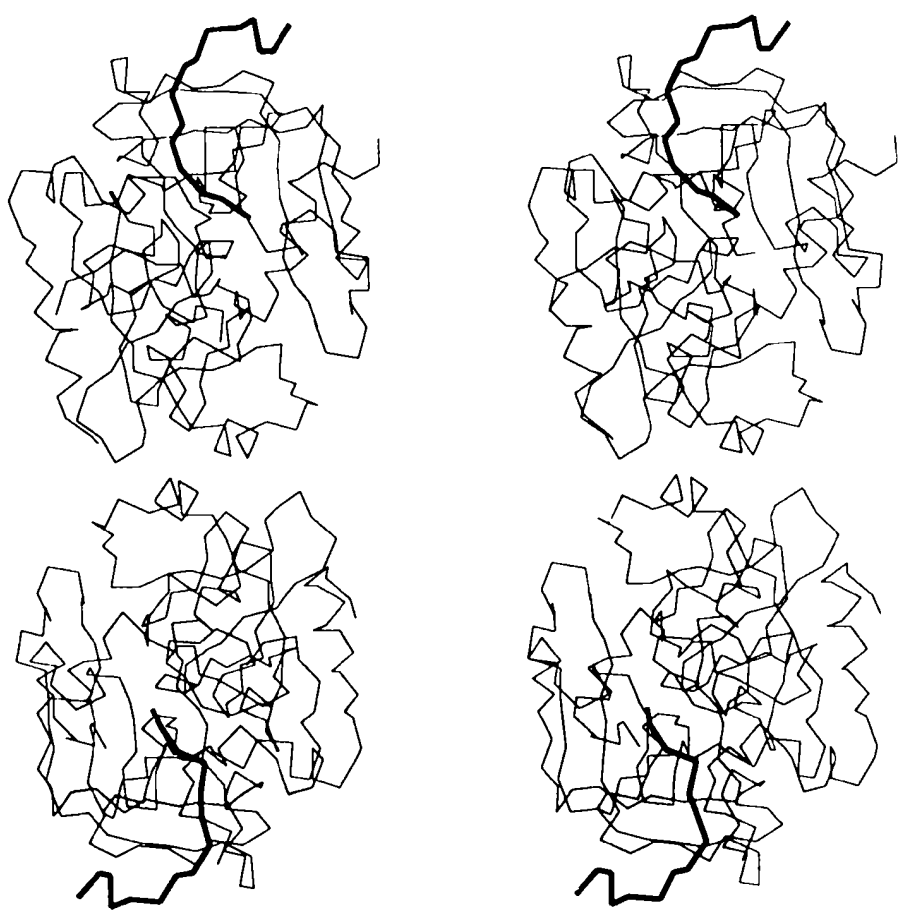

Fig. 4. A stereoview of the $\mathrm{C}_{\alpha}$ backbone of the two complexes looking down the approximate twofold axis that relates them. Complex 2 is on top of the figure. Thick lines are used for the peptides, thin lines for thrombin. The non-crystallographic rotation axis has spherical polar angles $\psi=69.0^{\circ} ; \dot{\varphi}=85.3^{\circ}$. 
Table 3. Electrostatic interactions between hirudin ${ }_{51-65}$ and bovine thrombin

\begin{tabular}{|c|c|c|c|c|}
\hline Residue & Interaction & Type & $\begin{array}{l}\text { Complex } 1 \\
(\dot{\mathrm{A}})\end{array}$ & $\begin{array}{c}\text { Complex } 2 \\
(\dot{\mathrm{A}})\end{array}$ \\
\hline Phe56h & $\mathrm{O} \leftarrow \mathrm{G} \ln 38 \mathrm{NE} 2$ & Hydrogen bond & 3.0 & \\
\hline \multirow{5}{*}{ Glu57h } & $\mathrm{N} \rightarrow \mathrm{Thr} 74 \mathrm{O}$ & Hydrogen bond & 3.4 & 3.1 \\
\hline & $\mathrm{OE} 1 \leftarrow$ Tyr $76 \mathrm{~N}$ & Hydrogen bond & 3.4 & $3.5^{*}$ \\
\hline & OE2 $\leftarrow$ Tyr76 N & Hydrogen bond & & $3.2 *$ \\
\hline & $\mathrm{OE} 2 \leftarrow \operatorname{Arg} 75 \mathrm{NH} 2$ & Ion pair/Hydrogen bond & 3.3 & \\
\hline & $\mathrm{OE} 2 \leftarrow \operatorname{Arg} 175^{\prime} \mathrm{NH} 1$ & Ion pair/Hydrogen bond & 2.9 & \\
\hline Glu58h & $\mathrm{OE} 1 \leftarrow \operatorname{Arg} 175^{\prime} \mathrm{NH} 1$ & Ion pair/Hydrogen bond & 3.3 & \\
\hline Glu61h & $\mathrm{OE} 1 \leftarrow \operatorname{Arg} 101^{\prime} \mathrm{NH} 1$ & Ion pair & 3.7 & \\
\hline
\end{tabular}

* The angle at the acceptor atom, which lies between $70-90^{\circ}$, is strained.

contribute (Stone, Betz, Parry, Jackman \& Hofsteenge, 1993; Yue et al., 1992). The interactions observed in the present structure agree with these results in that Glu57h forms hydrogen bonds and salt bridges with thrombin in complex 1 (Fig. 2) and hydrogen bonds in complex 2 (Fig. 3) whereas Glu58h, Glu61h and Glu62h do not interact directly with the thrombin molecule in either complex. As fit best to the electron density, Asp55h in complex 1 does not interact with thrombin although there is some density for an alternate position of the side chain that could salt bridge with Arg73. In complex 2 Asp55h makes a weak salt link with $\operatorname{Arg} 73(4.3 \AA)$. In bovine thrombin-hirudin, Asp55h has two weak salt links to $\operatorname{Arg} 73$ while in human thrombin-hirugen it forms two strong salt links. Similar observations in other thrombin-hirudin complexes have prompted the suggestion that the noninteracting acidic residues of the hirudin peptide contribute indirectly through a favourable interaction with a strong positive electrostatic field at the thrombin exosite (Karshikov et al., 1992).

The existence of the electrostatic interactions in the complexes under discussion (Table 3) is remarkable, given that they are crystallized at $1.6 \mathrm{M}$ ammonium sulfate. By contrast, bovine thrombin-hirudin (Vitali et al., 1992) and human thrombin-hirugen (SkrzypczakJankun et al., 1991) were crystallized from approximately 0.35 and $0.43 M$ salt, respectively, using polyethylene glycol as the precipitant. At zero ionic strength, approximately $1 / 3$ of the free energy of binding of hirudin to thrombin is electrostatic (Karshikov et al., 1992); the electrostatic free energy is halved when the ionic strength is increased to $0.125 \mathrm{M}$ while the contribution from other sources, such as van der Waals contacts and hydrophobic exclusion, remains unchanged (Karshikov et al., 1992). More generally, others have argued that surface salt bridges are not stable at physiological ionic strength because the energy gained in a salt link is approximately equal to the entropy lost when the side chains are restrained (Sali, Bycroft \& Fersht, 1991; Dao-Pin, Sauer, Nicholson \& Matthews, 1991). Consequently, stable surface links require supplemental interactions that overcome the entropy cost by fixing the residues near one another.
Here, the hydrophobic free energy, particularly from Phe56h, Ile59h, Pro60h, and Tyr63h in complex 1 and Phe56h and Tyr63h in complex 2 anchors the peptide chain, thereby fostering the electrostatic interactions.

The importance of the hydrophobic residues, Phe56h, Ile59h and Tyr63h, in the formation of the thrombinhirudin complex has been shown by mutagenesis (Stone et al., 1993; Yue et al., 1992). In both complexes reported here, the side chain of Phe56h is sandwiched between the ring of Phe 34 and Thr74, making close contacts with Phe34, Thr74 and Leu40 (Figs. 2 and 3). The stacking of the phenylalanine rings has the characteristic edge-on pattern (Burley \& Petsko, 1985). In complex 1, Ile59h, Pro60h and Tyr63h make close contacts with Leu65, Tyr76, and with Leu65 and Ile82 of thrombin, respectively, and with each other, while Tyr63h in complex 2 makes close contacts with Leu65 and Met84. Similar hydrophobic interactions are present in the bovine thrombin-hirudin complex (Vitali et al., 1992) and in the human thrombin-hirugen complex (Skrzypczak-Jankun et al., 1991).

Peptide 1 is better defined than peptide 2 because it has many more electrostatic interactions with thrombin, including some ion pairs that involve crystal packing contacts (Table 3). However, the greater disorder of peptide 2 is at least partly related to the cleavage at Thr149A in $\varepsilon$-thrombin, as evidenced by its lower affinity for hirudin compared to $\alpha$-thrombin in solution (Stone, Braun \& Hofsteenge, 1987). In complex 1, Thr149A and Ser149B in the intact $\gamma$-autolysis loop are within $3.5 \AA$ of peptide 1 and can potentially form hydrogen bonds with Asn52h and Asp53h, respectively. In complex 2 the two parts of the severed loop in $\varepsilon$ thrombin have moved away from the peptide in the exosite. This movement breaks any direct interactions, and should also perturb the electrostatic field.

These observations are also germane to the fact that hirudin (Dodt et al., 1988, 1990), and hirudin-related carboxyl-terminal fragments (Maraganore et al., 1989; DiMaio et al., 1990) bind more tightly to human than to bovine $\alpha$-thrombin because of species differences in the interaction at the exosite (Dodt et al., 1990). Human thrombin has alanine replacing Thr149A and asparagine replacing Ser149B. Previously, the only established 
difference occurred at position 149E, which is a lysine in human thrombin and a glutamate in bovine thrombin. In human thrombin, Lys $149 \mathrm{E}$ contributes to the positive electrostatic field at the exosite and forms an ion pair with Asp55h in the complex with hirudin (Rydel et al., 1991). In bovine thrombin, Glu149E can do neither (Vitali et al., 1992). The $\gamma$-autolysis loop, which is very mobile, has different conformations in different thrombin structures and is usually ordered only if stabilized by crystal contacts (Vijayalakshmi, Padmanabhan, Mann \& Tulinsky, 1994). Thus, the conformation of the $\gamma$-autolysis loop in complex 1 is not unique but does suggest how the loop could interact with exosite ligands in solution.

This work as supported in part by NIH grant GM 33192 and a fellowship for Dr Vitali from NIH training grant T32 HL07602. Coordinates and structure factors have been deposited in the Brookhaven Protein Data Bank.* The program $R H O M O D$ can be obtained from PDM (martin\% xray.dnet@rocdec.roc.wayne.edu).

\footnotetext{
* Atomic coordinates and structure factors have been deposited with the Protein Data Bank, Brookhaven National Laboratory (Reference: 1VIT, R1VITSF). Free copies may be obtained through The Managing Editor, International Union of Crystallography, 5 Abbey Square, Chester CH1 2HU, England (Reference: GR0441). At the request of the authors, the atomic coordinates will remain privileged until 1 January 1997 and the structure factors will remain privileged until 1 January 2000.
}

\section{References}

Abola, E. E., Bernstein, F. C., Bryant, S. H., Koetzle, T. F. \& Wang (1987). Crystallographic Databases, Information Content, Software Systems, Scientific Application, edited by F. H. Allen, G. Bergerhoff \& R. Sievers, pp. 107-132. Cambridge/Chester: IUCr.

Banner, D. W. \& Hadvary, P. (1991). J. Biol. Chem. 266, 20085-20093.

Bode, W., Mayr, I., Baumann, U., Huber, R., Stone, S. R. \& Hofsteenge, J. (1989). EMBO J. 8, 34673475 .

Bourdon, P., Jablonski, J.-A., Chao, B. H. \& Maraganore, J. M. (1991). FEBS Lett. 294, 163-166.

Brünger, A. T. (1988). J. Mol. Biol. 203, 803-816.

Brünger, A. T. (1990). Acta Cryst. A46, 46-57.

Burley, S. K. \& Petsko, G. A. (1985). Science, 229, 2328.

Butkowski, R. J., Elion, J., Downing, M. R. \& Mann, K. G. (1977). J. Biol. Sci. 252, 4942-4957.

Cambillau, C. \& Horjales, E. (1987). J. Mol. Graphics, 5, 174-177.

Clore, G. M., Sukermaran, D. K., Nilges, M., Zarbock, J. \& Gronenborn, A. M. (1987). EMBO J. 6, 529-539.

Coughlin, S. R. (1994). Trends Cardiovascular Med. 4, 77-83.
Dao-Pin, S., Sauer, U., Nicholson, H. \& Matthews, B. W. (1991). Biochemistry, 30, 7142-7153.

Davie, E. W., Fujikawa, K. \& Kisiel, W. (1991). Biochemistry, 30, 10363-10370.

Degan, S. J. F., MacGillivray, R. T. A. \& Davie, E. W. (1983). Biochemistry, 22, 2087-2097.

DiMaio, J., Gibbs, B., Munn, D., Lefebvre, J., Ni, F. \& Konishi, Y. (1990). J. Biol. Chem. 265, 2169821703.

Dittman, W. A. \& Majerus, P. W. (1990). Blood, 75, 329336.

Dodt, J., Kohler, S. \& Baici, A. (1988). FEBS Lett. 229, 87-90.

Dodt, J., Kohler, S., Schmitz, T. \& Wilhelm, B. (1990). J. Biol. Chem. 265, 713-718.

Engh, R. A. \& Huber, R. (1991). Acta Cryst. A47, 392400.

Esmon, C.T. (1987). Science, 235, 1348-1352.

Fenton, J. W. II. (1981). Ann. NY Acad Sci. 370, 468495.

Fenton, J. W. II. (1986). Ann. NY Acad. Sci. 485, 5-15.

Folkers, P. J. M., Clore, G. M., Driscoll, P. C., Dodt, J., Kohler, S. \& Gronenborn, A. M. (1989). Biochemistry, 28, 2601-2617.

Furey, W., Wang, B. C. \& Sax, M. (1982). J. Appl. Cryst. 15, 160-166.

Grütter, M. G., Priestle, J. P., Rahuel, J., Grossenbacher, H., Bode, W, Hofsteenge, J. \& Stone, S. R. (1990). EMBO J. 9, 2361-2365.

Haruyama, H. \& Wüthrich, K. (1989). Biochemistry, 28, 4301-4312.

Howard, A. J., Gilliland, G. L., Finzel, B. C., Poulos, T. L., Ohlendorf, D. H. \& Salemme, F. R. (1987). J. Appl. Cryst. 20, 383-387.

Johnson, P. H., Sse, P., Winant, R., Hudson, D., Underhill, P., Lazar, J. B, Olsen, C. \& Almquist, R. (1991). Haemostasis, 21, 41-48.

Jones, T. A. (1985). Methods Enzymol. 115, 157-171.

Karshikov, A., Bode, W., Tulinsky, A. \& Stone, S. R. (1992). Protein Sci. 1, 727-735.

Laskowski, R. A. MacArthur, M. W. \& Thornton, J. M. (1994). Evaluation of Protein Coordinate Sets. In From First Map to Final Model, edited by S. Bailey, R. Hubbard, \& D. A. Waller, pp. 149-159. Warrington: Daresbury Laboratory.

MacGillivray, R. T. A. \& Davie, E. W. (1984). Biochemistry, 23, 1626-1634.

Magnusson, S., Petersen, T. E., Sottrup-Jensen, L. \& Claeys, H. (1975). Proteases and Biological Control, edited by E. Reich, D. B. Rifkin \& E. Shaw, pp. 123-149. Cold Spring Harbor Laboratory Press.

Mann, K. G. (1987). Trends Biochem. Sci. 12, 229233.

Maraganore, J. M., Bourdon, P., Jablonski, J., Ramachandran, K. L. \& Fenton, J. W., II (1990). Biochemistry, 29, 7095-7101.

Maraganore, J. M., Chao, B., Joseph, K. L., Jablonski, J. \& Ramachandran, K. L. (1989). J. Biol. Chem. 264, 86928698.

Markwardt, F. (1970). Methods Enzymol. 19, 924-932.

Martin, P. D., Kumar, V. K., Tsernoglou, D. \& Edwards, B. F. P. (1983). Fed. Proc. 42, 1861. 
Maryanoff, B. E., Qiu, X., Padmanabhan, K. P., Tulinsky, A., Almond H. R., Andrade-Gordon, P., Greco, M. N., Kauffman, J A., Nicolaou, K. C., Liu, A., Brungs, P. H., \& Fusetani, N. (1993). Proc. Natl Acad. Sci. USA, 90, 8048-8052.

Priestle, J. P., Rahuel, J., Rink, H., Tones, M. \& Grütter, M. G. (1993). Protein Sci. 2, 1630-1642.

Qiu, X., Padmanabhan, K. P., Carperos, V. E., Tulinsky, A., Kline, T., Maraganore, J. M. \& Fenton, J. W. (1992). Biochemistry, 31, 11689-11697.

Read, R. J. (1986). Acta Cryst. A42, 140-149.

Rossman, M. G. \& Blow, D. M. (1962). Acta Cryst. 15, 24-31.

Rydel, T. J., Ravichandran, K. G., Tulinsky, A., Bode, W., Huber, R., Roitsch, C. \& Fenton, J. W. (1990). Science, 249, 277-290.

Rydel, T. J., Tulinsky, A, Bode, W. \& Huber, R. (1991). J. Mol. Biol. 221, 583-601.

Sali, D., Bycroft, M. \& Fersht, A. R. (1990). J. Mol. Biol. 220, 779-788.

Satow, Y., Cohen, G. H., Padlan, E. A. \& Davies, D. R. (1986). J. Mol. Biol. 190, 593-604.

Skrzypczak-Jankun, E., Carperos, V. E., Ravichandran, K. G., Tulinsky, A, Westbrook, M. \& Muraganore, J. M. (1991). J. Mol. Biol. 221, 1379-1393.

Stone, S. R., Braun, P. J. \& Hofsteenge, J. (1987). Biochemistry, 26, 4617-4624.
Stone, S. R., Betz, A., Parry, M. A. A., Jackman, M. P. \& Hofsteenge, J. (1993). Exp. Med. Biol. 340, 35-49.

Stubbs, M. T., Oschkinat, H., Mayr, I., Huber, R, Angliker, H., Stone, S. R. \& Bode, W. (1992). Eur. J. Biochem. 206, 187-195.

Vijayalakshmi, J., Padmanabhan, K. P., Mann, K. G. \& Tulinsky, A. (1994). Protein Sci. 3, 2254-2271.

Vitali, J., Martin, P. D., Malkowski, M. G., Robertson, W. D., Lazar, J. B, Winant, R. C., Johnson, P. H. \& Edwards, B. F. P. (1992). J. Biol. Chem. 267, 1767017678.

Walz, D. A., Hewett-Emmett, D. \& Guillin, M.-C. (1986). Prothrombin and Other Vitamin K Proteins, edited by W. H. Seegers \& D. A. Walz, Vol. 1, pp. 125-160. Boca Raton, FL: CRC Press.

Wu, T.-P., Yee, V., Tulinsky, A., Chrusciel, R. A., Nakanishi, H., Shen, R., Priebe, C. \& Kahn, M. (1993). Protein Eng. 6, 471-478.

Yue, S.-Y., DiMaio, J., Szewczuk, Z., Purisima, E. O., Ni, F. \& Konishi, Y. (1992). Protein Eng. 5, 7785

Zdanov, A., Wu., S., DiMaio, J., Konishi, Y., Li, Y., Wu, X., Edwards, B. F. P., Martin, P. D. \& Cygler, M. (1993). Proteins Struct. Funct. Genet. 17, 252-265.

Zhang, K. Y. J. (1993). Acta Cryst. D49, 213-222.

Zhang, X.-J. \& Matthews, B. W. (1994). Acta Cryst. D50, 675-686. 